\title{
Chaperones as Suppressors of Protein Misfolded Oligomer Toxicity
}

\author{
Benedetta Mannini ${ }^{1}$ and Fabrizio Chiti ${ }^{2 *}$ \\ ${ }^{1}$ Department of Chemistry, University of Cambridge, Cambridge, UK, ${ }^{2}$ Section of Biochemistry, Department of Experimental \\ and Clinical Biomedical Sciences, University of Florence, Florence, Italy
}

Chaperones have long been recognized to play well defined functions such as to: (i) assist protein folding and promote formation and maintenance of multisubunit complexes; (ii) mediate protein degradation; (iii) inhibit protein aggregation; and (iv) promote disassembly of undesired aberrant protein aggregates. In addition to these well-established functions, it is increasingly clear that chaperones can also interact with aberrant protein aggregates, such as pre-fibrillar oligomers and fibrils, and inhibit their toxicity commonly associated with neurodegenerative diseases without promoting their disassembly. In particular, the evidence collected so far in different labs, exploiting different experimental approaches and using different chaperones and client aggregated proteins, indicates the existence of two distinct mechanisms of action mediated by the chaperones to neutralize the toxicity of aberrant proteins oligomers: (i) direct binding of the chaperones to the hydrophobic patches exposed on the oligomer/fibril surface, with resulting shielding or masking of the moieties responsible for the aberrant interactions with cellular targets; (ii) chaperone-mediated conversion of aberrant protein aggregates

OPEN ACCESS

Edited by: Angelo Poletti, Università degli Studi di Milano, Italy

Reviewed by: Stephane Pelletier, St. Jude Children's Research Hospital, USA Leonidas Stefanis, University of Athens Medical School,

Greece

*Correspondence: Fabrizio Chiti fabrizio.chiti@unifi.it

Received: 24 January 2017 Accepted: 23 March 2017

Published: 05 April 2017

Citation:

Mannini $B$ and Chiti F (2017) Chaperones as Suppressors

of Protein Misfolded

Oligomer Toxicity.

Front. Mol. Neurosci. 10:98. doi: 10.3389/fnmol.2017.00098 into large and more innocuous species, resulting in a decrease of their surface-to-volume ratio and diffusibility and in deposits more easily manageable by clearance mechanisms, such as autophagy. In this review article we will describe the in vitro and in vivo evidence supporting both mechanisms and how this results in a suppression of the detrimental effects caused by protein misfolded aggregates.

Keywords: proteostasis, protein misfolding diseases, clustering of aggregates, oligomer toxicity, aggresomes, inclusion bodies, amyloid fibrils, neurodegeneration

\section{INTRODUCTION}

The various proteins that constitute the human proteome are functional if they fold correctly, remain soluble, can be trafficked properly, form functional complexes and perform their task correctly. These abilities rely on the existence of a proteostasis network and on its proper running. The proteostasis network is constituted by the translational machinery, a large body of molecular chaperones and co-chaperones, the autophagy/lysosome system (ALS) and the ubiquitin/proteasome system (UPS). Molecular chaperones and co-chaperones can be grouped in distinct families, including ribosome-binding chaperones, Hsp40s, Hsp70s, chaperonins, Hsp90s, Hsp100, prefoldins, small heat shock proteins (sHsps) and TPR-domain containing co-chaperones. It has been estimated that this large body of proteins amounts to $c a .330$ distinct polypeptide chains in the human proteome (Brehme et al., 2014).

Molecular chaperones have long been recognized to: (i) assist protein folding from unfolded or partially folded states and promote formation and maintenance of multisubunit complexes; 
(ii) mediate protein degradation via the UPS or ALS systems; (iii) inhibit protein aggregation by binding to fully or partially unfolded states; and (iv) promote disassembly of undesired aberrant protein aggregates (Kaushik and Cuervo, 2012; Labbadia and Morimoto, 2015; Mogk et al., 2015; Balchin et al., 2016). Although this last function has long been known to involve only chaperones of the $\mathrm{ClpB}$ and Hsp104 families present in bacteria, protozoa, plants and fungi (Mogk et al., 2015), it has recently been discovered in higher eukaryotes as well (Nillegoda and Bukau, 2015; Nillegoda et al., 2015).

However, in addition to these four well established functions, evidence is mounting that molecular chaperones can also interact with protein aggregates (macromolecular species resulting from the aberrant self-assembly of proteins) and inhibit their toxicity without promoting their disassembly. In this review we will show the evidence in this direction, showing how molecular chaperones can interact with both protein oligomers and amyloid fibrils, that represent the small species forming at the beginning of the aggregation process and the fibrillar end-product of this phenomenon, respectively. We will describe the mechanism through which molecular chaperones suppress the detrimental effects of such aggregates in the absence of their disaggregation or clearance.

\section{CHAPERONES BIND TO PROTEIN OLIGOMERS}

An increasing number of reports has shown the ability of molecular chaperones to interact with oligomeric species that form early during the aggregation of proteins. The extracellular chaperone clusterin has been observed, through Thioflavin $\mathrm{T}$ (ThT) kinetics and dot blot assays, to bind to prefibrillar species formed by six different amyloidogenic proteins or peptides at a substoichiometric 1:10 molar ratio (Yerbury et al., 2007). Using transmission electron microscopy (TEM), ThT kinetics and size exclusion chromatography (SEC), Hsp104 was found to bind to $A \beta_{42}$ oligomers and protofibrils, but also to small fibrils, and abolish their ability to convert into amyloid fibrils through their further addition to preformed fibrils, as well as to abrogate their capacity to seed the addition of monomers to the fibril surface, up to a Hsp104:A $\beta_{42}$ stoichiometric ratio of 1:1000 (Arimon et al., 2008). By means of single-molecule fluorescence methods, clusterin (Narayan et al., 2011) and the sHsp $\alpha$ B-crystallin or HSPB5 (Narayan et al., 2012) were observed to form longlived, stable complexes with $A \beta_{40}$ oligomers at equimolar ratios. Such sequestration was found also in the case of $\alpha \mathrm{B}$-crystallin and SOD1 aggregates, using ThT kinetics and SEC coupled with SDS-PAGE, with $\alpha \mathrm{B}$-crystallin:SOD1 molar ratios of 1:100 and 1:1, respectively (Yerbury et al., 2013). A quantitative kinetic analysis and immunochemistry studies revealed that the chaperone DNAJB6, from the Hsp40 family, preferentially binds to oligomeric species of $A \beta_{42}$ at low substoichiometric molar ratios (up to 1:200), preventing their growth into longer fibrils as well as the formation of new fibril nuclei (Månsson et al., 2014).

This ability of chaperones to bind to protein oligomers is not just aimed at interfering with the process of amyloid fibril formation, but has also the important effect to inhibit directly the toxicity of these aberrant species. In fact, oligomers are characterized by physicochemical properties that make them harmful to cells (Campioni et al., 2010; Olzscha et al., 2011; Bemporad and Chiti, 2012). Among the structural determinants of toxicity identified so far, the small size and the high extent of hydrophobic surface of the oligomers have been recognized to play an important role in causing cellular dysfunction (Bolognesi et al., 2010; Cizas et al., 2010; Mannini et al., 2014). It is just on these structural determinants that molecular chaperones seem to act with the goal of counteracting the damages mediated by the oligomers: in fact chaperones increase the size of the oligomers and mask hydrophobic patches exposed on their surface. Such evidence will be described in the next two sections.

\section{CHAPERONES INDUCE CLUSTERING OF THE OLIGOMERS AND INHIBIT THEIR TOXICITY}

In an early report, the ability of clusterin to inhibit the toxicity of preformed oligomers of $A \beta_{42}$ and of the SH3 domain of phosphatidylinositol 3-kinase (PI3-SH3) was observed on human neuroblastoma SH-SY5Y cells at 1:10 clusterin:substrate ratio (Yerbury et al., 2007). Although the mechanism of action of the chaperone against the aggregates was not investigated in detail, the authors reported a sedimentation assay in which the formation of high molecular weight $A \beta_{42} /$ clusterin complexes were observed (Yerbury et al., 2007).

Later on, Hsp27 (HSPB1) was found to increase the size of preformed $A \beta_{42}$ oligomers, making them unable to exert their toxicity on N2a mouse neuroblastoma cell cultures (Ojha et al., 2011). In particular, atomic force microscopy (AFM), TEM and light scattering showed that the incubation of a substoichiometric concentration of Hsp27 with preformed $A \beta_{42}$ oligomers (1:5) in vitro generates larger aggregates. In these larger species Hsp27 co-precipitated with the $A \beta_{42}$ oligomers, without affecting significantly the structure of the oligomers, as shown by unaltered ThT binding and far-UV circular dichroism (CD) spectra. Interestingly, even the ability to bind to 8-anilinonaphthalene-1-sulfonic acid (ANS) was unalterd, indicating that the extent of the hydrophobic surface exposure does not change after the incubation with the chaperone.

In another study, five different chaperones, namely, $\alpha$ B-crystallin, Hsp70 (HSPA1A), clusterin, $\alpha_{2}$-macroglobulin and haptoglobin were able to suppress the toxicity of oligomers formed by three different proteins, the $A \beta_{42}$ peptide, the islet amyloid polypeptide (IAPP), and the model protein HypF-N at a substoichiometric concentration (up to 1:1000) on SH-SY5Y cells (Mannini et al., 2012). Several methods of investigation applied to the HypF-N system, such as AFM, confocal microscopy coupled to immunostaining, centrifugation assays and intrinsic fluorescence, showed that large clusters of HypF-N oligomers are formed following the incubation with substoichiometric concentrations of chaperones. In the resulting large aggregates, chaperone molecules are trapped inside the 
large aggregates. Again, the chaperone-mediated conversion of the small oligomers into bigger nontoxic aggregates occurs without a remodeling of the structure of the oligomers, as assessed by Fourier-transform infrared spectroscopy and pyrene labeling (Mannini et al., 2012).

Recently, it was found that high amounts of insoluble aggregates containing chaperones, in particular the sHsps, accumulate in the long-lived daf- 2 mutants of the nematode Caenorhabditis elegans during aging, and that such amounts are higher compared to the wild-type controls that had a normal lifespan (Walther et al., 2015). It was suggested that the chaperones neutralize aberrant, potentially toxic, proteins and soluble oligomers by driving them into insoluble large aggregates and that this strategy enables to slow down the decline of the proteostasis network during normal aging and extend the lifespan of the mutant nematodes (Walther et al., 2015).

A similar mechanism was observed on various cell culture systems, such as $\mathrm{CHO}, \mathrm{N} 2 \mathrm{a}, \mathrm{NIH}-3 \mathrm{~T} 3, \mathrm{PC} 12$ and MEF, treated with NT219, an inhibitor of the insulin/IGF-1 signaling pathway (Moll et al., 2016). NT219 was found to enhance the aggregation of misfolded prion proteins and promote its deposition in intracellular inclusions such as the aggresomes. Although NT219 was also found to increase the concentrations of certain molecular chaperones, it also reduces proteasome activity and impairs autophagy, indicating that conversion of proteins into large aggresomes is a protective mechanism even in the absence of their immediate clearance (Moll et al., 2016).

\section{CHAPERONES SHIELD THE HYDROPHOBIC MOIETIES OF THE OLIGOMERS AND INHIBIT THEIR TOXICITY}

The chaperone-induced clustering of aberrant protein oligomers is not the only mechanism through which these important protein molecules protect against oligomer toxicity. Evidence has been shown, using surface plasmon resonance (SPR), on the ability of clusterin to bind to toxic $A \beta_{42}$ oligomers at substoichiometric ratio (1:1000) and shield their reactive hydrophobic patches on their surface (Beeg et al., 2016). The pre-incubation of these aggregates with clusterin also reverted their ability to reduce the pharyngeal mobility in C. elegans nematodes (Beeg et al., 2016). In another study, Hsp70 was modified to be released in the extracellular space in order to address its protective activity against $\mathrm{A} \beta_{42}$ in Drosophila melanogaster models (Fernandez-Funez et al., 2016). The secreted form, called secHsp70, suppressed $A \beta_{42}$ toxicity, as deduced by decreased eye degeneration, reduced neuronal death, structural integrity of adult neurons, suppression of locomotor neuron dysfunction, and lifespan extension. An assay based on luciferase-derived luminescence showed that secHsp70 stabilizes $A \beta_{42}$ oligomeric species and masks their neurotoxic epitopes, thus promoting the accumulation of nontoxic aggregates (Fernandez-Funez et al., 2016). This activity was carried out in the absence of ATP, indicating therefore that secHsp70 exploits its holdase function to interact with the oligomers in the absence of detectable clustering (FernandezFunez et al., 2016). It is interesting to note that other Hsps that were found able to interact with aggregates, such as Hsp104 (Arimon et al., 2008; Castellano et al., 2015), Hsp70 (Mannini et al., 2012) and Ssalp (Xu L. et al., 2013), exert the capacity to bind to the aggregates without consumption of ATP.

Stabilization of oligomeric $A \beta_{42}$ has also been observed in the presence of human prefoldin (hPFD) at substoichiometric molar ratio (up to 1:500) using western blot and TEM. Viability assays on cultured PC12 cells or primary cortical neurons from embryonic mice show that the $A \beta_{42} / \mathrm{hPFD}$ complexes are less toxic than complexes of similar size obtained by incubating $A \beta_{42}$ oligomers with archaeal prefoldin (PhPFD). The different biological activity was attributed to the higher hydrophobic exposure and $\beta$-sheet content of $A \beta_{42} / \mathrm{PhPFD}$ complexes, as assessed by ANS and ThT binding (Sörgjerd et al., 2013).

These observations highlight the existence of different mechanisms of action mediated by the chaperones against the toxicity of the oligomers, that is "binding followed by clustering" and "binding causing hydrophobic shielding in the absence of clustering". Both mechanisms have been indeed observed on a recent report in which the effect of the chaperones $\alpha \mathrm{B}$-crystallin and clusterin, and an engineered monomeric variant of transthyretin known to have a chaperone-like activity, was investigated over a wide range of concentrations, both superand sub-stoichiometric relative to HypF-N toxic oligomers, ranging from 4:1 to 1:16 (Cappelli et al., 2016). AFM images and light scattering measurements showed that the chaperones increase the size of the aggregates to an extent that correlates with chaperone concentration, ranging from null to remarkable increase. Notably, the protective effect on N2a cells was observed at all chaperone concentrations, irrespective of the size increase. Measurements of ANS binding showed that in the large clusters the overall exposure of the hydrophobic surface does not change, whereas when the clustering promoted by the chaperones is negligible the ANS binding is reduced, indicating that the hydrophobicity on the surface is shielded by the chaperones.

\section{CHAPERONES BIND TO AMYLOID FIBRILS}

Prefibrillar oligomers are not the only molecular target of molecular chaperones. An increasing body of reports also describe the ability of these guardian proteins to bind to mature fibrils. In this regard, the $\mathrm{sH} p \alpha \mathrm{B}$-crystallin has been extensively studied because it colocalizes with $\mathrm{A} \beta$ plaques (Shinohara et al., 1993) and Lewy bodies (Wakabayashi et al., 2007), that are the neuropathological hallmarks of Alzheimer's disease and Parkinson's disease, respectively. Indeed, this chaperone has been found to bind to fibrils from $A \beta_{40}$ (Raman et al., 2005), $\mathrm{A} \beta_{42}$ and the Arctic variant E22G A $\beta_{42}$ (Shammas et al., 2011), and $\alpha$-synuclein (Waudby et al., 2010). The binding prevents fibril growth of $\mathrm{A} \beta_{40}$, as revealed by ThT binding assays, 
total reflection fluorescence microscopy and CD measurements (Raman et al., 2005). A strong inhibition of fibril growth was also demostrated for $A \beta_{42}$ (Shammas et al., 2011) and $\alpha$-synuclein (Waudby et al., 2010), by measurements of seeded fibril elongation kinetics, both in solution and on the surface of a quartz crystal microbalance (QCM). Immunoelectron microscopy images showed that $\alpha \mathrm{B}$-crystallin binds along the entire lenght and ends of the $A \beta_{42}$ (Shammas et al., 2011) and $\alpha$-synuclein fibrils (Waudby et al., 2010). Although cell toxicity measurements were not reported directly, it was hypothesized that the binding of $\alpha \mathrm{B}$-crystallin to fibrils may reduce their toxicity by shielding the exposed hydrophobic residues and by preventing the generation of new oligomers that occurs on the fibril surface due to secondary nucleation (Waudby et al., 2010).

The ability of $\alpha \mathrm{B}$-crystallin to bind to fibrillar species and inhibit their growth has also been observed with many other non-neuronal amyloid fibril systems, such as insulin fibrils at low pH (Knowles et al., 2007), $\beta_{2}$-microglobulin $\left(\beta_{2}-\mathrm{m}\right)$ fibrils at low pH (Raman et al., 2005) and apolipoprotein C-II (apoC-II) fibrils at neutral pH (Binger et al., 2013). Interestingly, by exploiting the property of $\beta_{2}-\mathrm{m}$ fibrils to depolymerize when shifted to neutral $\mathrm{pH}$, it was found that $\alpha \mathrm{B}$-crystallin retards fibril depolymerization (Raman et al., 2005). A chaperone-induced stabilization effect was also observed in the case of apoC-II fibrils, when their fragmentation promoted by dilution was inhibited in the presence of the chaperone (Binger et al., 2013). In the same report, $\alpha \mathrm{B}$-crystallin was also found, using TEM images and sedimentation assays, to induce the formation of large fibrillar tangles. Although the authors did not provide direct experimental evidence, they argued that these stabilized clumped inclusions represent a protective strategy because they are unable to release cytotoxic oligomers and to promote events of secondary nucleation (Binger et al., 2013).

A direct proof of the beneficial effect of the binding of chaperones to fibrils was obtained for the human Brichos domain (Cohen et al., 2015). This chaperone binds to the surface of $\mathrm{A} \beta_{42}$ fibrils, where the formation of oligomeric intermediates is catalyzed, and therefore minimizes the formation of toxic species, as demonstrated by several techniques, such as ThT kinetic analysis, TEM coupled with immunogold labeling, SPR and SEC in cojunction with immunoblot. Electrophysiology experiments in living mouse brain tissue, as well as cell viability measurements based on the MTS assay and the capsase- 3 activity quantification on SH-SY5Y cultured cell lines, verified that this mechanism effectively suppresses the oligomer-mediated damage.

\section{THE ABILITY OF OTHER NON-CHAPERONE PROTEINS TO BIND TO PROTEIN AGGREGATES AND INHIBIT THEIR TOXICITY}

Other proteins that are generally not classified as chaperones have been recognized to bind to aggregates and suppress their toxic effects, thus acting as officially recognized molecular chaperones. Soluble collagen VI was found to rescue mice neocortical/hippocampal neurons from the toxicity mediated by $A \beta_{42}$ oligomers by altering the interaction of the oligomers with neurons (Cheng et al., 2009). Indeed, immunostained confocal microscopy images showed that collagen VI prevents the association of $A \beta_{42}$ oligomers with the surface of cultured neurons and was found to colocalize with $A \beta_{42}$ into large deposits in the extracellular space, with the latter finding being confirmed by AFM. This mechanism of sequestration was found to result in a lower amount of soluble toxic oligomers in the extracellular space, with lack of binding to the neuron surface and protection from the damage (Cheng et al., 2009).

This protective strategy has also been observed, by means of immunolabeled confocal microscopy images, for the complement protein $\mathrm{C} 1 \mathrm{q}$, shown to prevent the association of fibrillar $A \beta_{42}$ to cultured mouse primary cortical neurons and to increase the size of $A \beta_{42}$ oligomeric species (Benoit et al., 2013). In particular, the accumulation of the oligomers into large deposits in the extracellular space impedes their internalization in the neurons, as demonstrated by the lower colocalization between the oligomers with a lysosomial marker in samples treated with C1q (Benoit et al., 2013).

Interesting is the case of transthyretin, which has been recently found to possess a generic ability to deal with protein misfolded oligomers (Li et al., 2011, 2013; Cascella et al., 2013). When pre-incubated with two different oligomeric species formed by the $A \beta_{42}$ peptide and the HypF-N protein, human tetrameric transthyretin (hTTR) and its engineered monomeric variant (M-TTR) effectively suppress their toxicity on SH-SY5Y cell cultures, again promoting the clusterization of the oligomers into larger species, as shown by AFM and immunostained confocal microscopy, in the absence of their structural reorganization, as shown by ThT fluorescence and the patterns of the pyrene spectra (Cascella et al., 2013).

The maltose binding protein (MBP) from Escherichia coli has been shown to induce the formation of bigger clusters of preformed $A \beta_{42}$ oligomers, visible in TEM images, which have a reduced perniciouness to SH-SY5Y cell cultures (Sharoar et al., 2013). These assamblies were found to display a lower level of ThT binding and $\beta$-sheet content in the CD spectra, indicating in this case a change in the secondary structure induced by MBP. Finally, the protein $\alpha_{\mathrm{s} 1}$-casein from bovine milk was found to inhibit fibril generation of $A \beta_{40}$ by redirecting the process towards the formation of amorphous aggregates (Carrotta et al., 2012). AFM images and data obtained with CD spectroscopy and ThT binding assays showed that $\alpha_{s 1}$-casein affects the formation of the oligomers and the growth of the protofibrils. The aggregates formed in its presence have a lower content in fibrillar structure and a large globular appearance (Carrotta et al., 2012).

Following all this experimental evidence it is clear that many proteins cooperate with molecular chaperones in defending the cells from the insults caused by aberrant protein oligomers and that a widespread proteostasis control exists, with a multiplicity of guardians in vivo. 


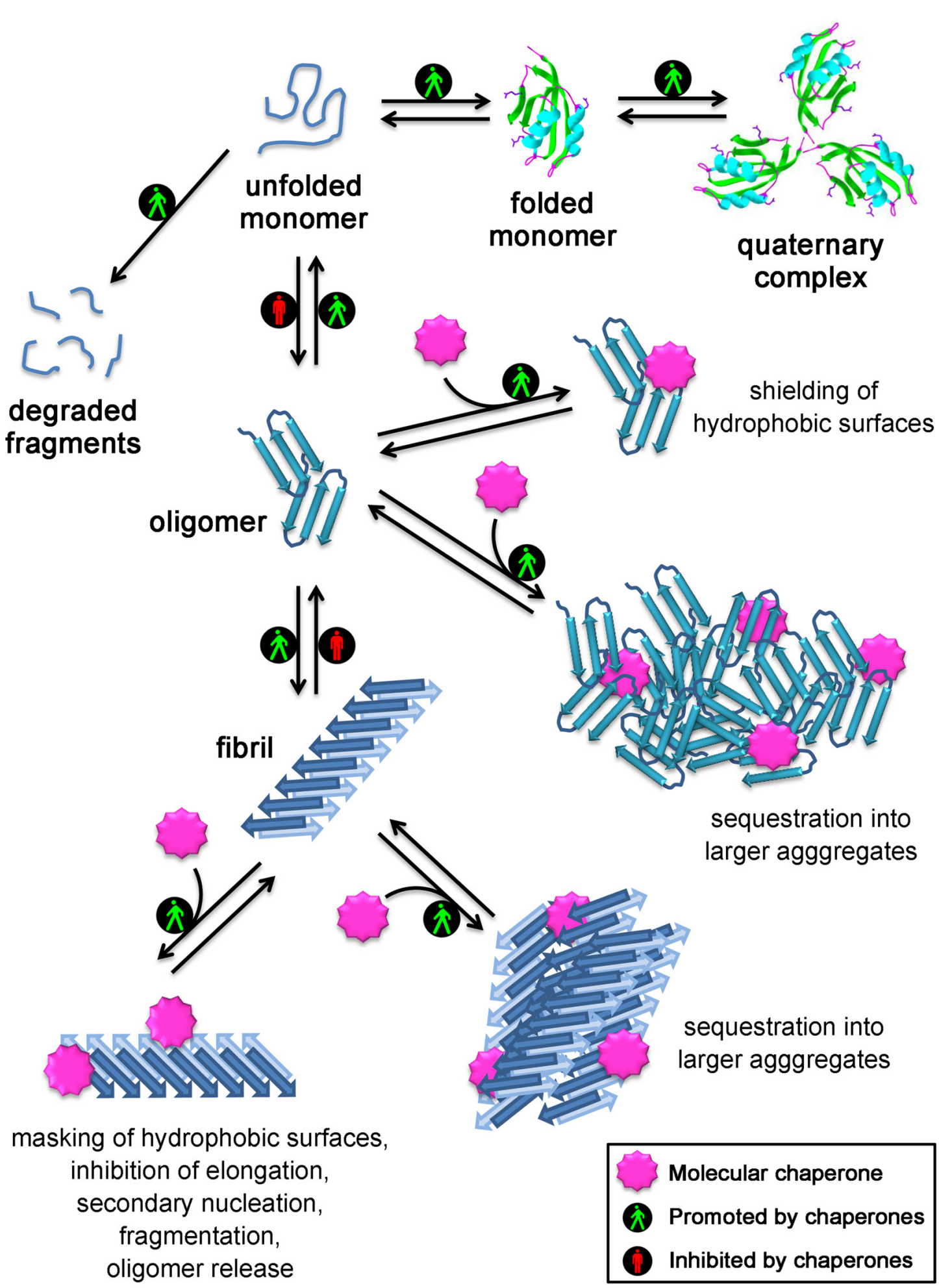

FIGURE 1 | Mechanisms used by molecular chaperones to maintain proteins in their soluble states and reduce the toxicity of protein aggregates. Chaperones assist protein folding, promote formation and maintenance of multisubunit complexes, mediate protein degradation, inhibit protein aggregation and promote disassembly of undesired protein aggregates. In addition, several strategies are employed by molecular chaperones to reduce the toxicity of protein aggregates: they act on small soluble oligomers by shielding their hydrophobic patches or by sequestering them into larger aggregates; they also promote the clustering of the fibrils, inhibit their elongation, the generation of oligomers through secondary nucleation occurring on the fibril surface, their fragmentation/oligomer release, and mask the reactive hydrophobic residues exposed on the fibril surface. 


\section{ASSEMBLY OF PROTEIN OLIGOMERS AND FIBRILS INTO LARGE AGGREGATES IN VIVO}

The neutralization of protein misfolded oligomers and fibrils following their binding to molecular chaperones and subsequent clustering into larger aggregates observed in vitro has also been found in vivo. It has been shown that in all living organisms, from bacteria to high eukaryotes, the aggregates formed intracellularly are assembled together in one or a limited number of inclusions (Villaverde and Carrió, 2003; Hyttinen et al., 2014; Miller et al., 2015), which are termed aggresomes in mammalian cells and are in close proximity to the centrosome (Johnston et al., 1998; Hyttinen et al., 2014).

The formation of the aggresome is thought to be a protective process able to sequester harmful aggregates and to act as a storage center for eventual degadation via authophagy (Villaverde and Carrió, 2003; Hyttinen et al., 2014; Miller et al., 2015). The assembly of small aggregates into the large aggresome is not mediated by a single chaperone, as observed in simplified experiments in vitro, but is a finely regulated process mediated by a complex machinery: misfolded aggregates are polyubiquitinated and associate with the microtbule-associated protein dynein, which transports them to the microtubule organizing center (MTOC) to merge them into the growing aggresome (Hyttinen et al., 2014). The association of the polyubiquitinated aggregate to the microtubule/dynein complex is mediated by chaperones of the Hsp70 family, the Hsp70 co-chaperone Bcl-2-associated athanogene 3 (BAG-3) and the protein 14-3-3 which has binding sites for both BAG-3 and dynein (Xu Z. et al., 2013; Jia et al., 2014). It can also be mediated by histone deacetylase 6 (HDAC6), which has binding sites for both ubiquitin and dynein (Kawaguchi et al., 2003; Ouyang et al., 2012). Importantly, however, the outcome is similar to that observed in vitro, that is neutralization of diffusible and potentially harmful oligomers into an innocuous and easily manageable large aggregate, which will later be degraded via autophagy.

Formation of large inclusion bodies has also been widely studied in yeasts, where three distinct inclusion bodies have been observed, namely the cytosolic quality control bodies (Q-bodies or cytoQ), the intranuclear quality control compartment (INQ, previously termed JUNQ) and the insoluble protein deposit (iPOD) forming close to the vacuole (Miller et al., 2015). Although the molecular mechanisms underlying formation of such inclusions are still largely unclear, it has been shown that

\section{REFERENCES}

Arimon, M., Grimminger, V., Sanz, F., and Lashuel, H. A. (2008). Hsp104 targets multiple intermediates on the amyloid pathway and suppresses the seeding capacity of A $\beta$ fibrils and protofibrils. J. Mol. Biol. 384, 1157-1173. doi: 10.1016/j.jmb.2008.09.063

Balchin, D., Hayer-Hartl, M., and Hartl, F. U. (2016). In vivo aspects of protein folding and quality control. Science. 353:aac4354. doi: 10.1126/science. aac 4354
cytoQ and INQ form from the fusion of smaller aggregates and that their formation is mediated by chaperones such as the small heat shock protein Hsp42 and the heat shock protein Btn2, respectively (Miller et al., 2015).

All the results that have shown the ability of molecular chaperones to interact with protein oligomers and neutralize their deleterious effects have mainly been obtained in cultured cell models. As described above, there is evidence that chaperones also induce formation of large aggregates in vivo, but the molecular mechanism by which this occurs and how such a complex tissue as the human brain benefits from this possibly protective process awaits specific experimental studies.

\section{CONCLUSIONS}

Overall, all the experimental evidence collected so far from both in vitro and in vivo studies indicate that chaperones do not just maintain proteins in their soluble native states, as thought until 5 years ago, but also directly bind to protein oligomers and fibrils and neutralize their deleterious effects. This may occur through: (i) the direct binding to the hydrophobic patches exposed on the oligomer/fibril surface, which are responsible for the aberrant interactions with a number of targets in the cell and on the cell membrane; or (ii) their conversion into large and more innocuous species, which appear to minimize their surface-tovolume ratio, their diffusibility and to be more easily manageable by clearance mechanisms, such as autophagy. A schematic representation of the mechanisms used by molecurar chaperones to maintain protein homeostasis is shown in Figure 1. In the light of the fact that the formation of large aggregates in the cells has a protective role, it is conceivable that the large size and spatially circumscribed nature of the histopathological signatures of various neurodegenerative diseases, such as the amyloid plaques and neurofibrillarly tangles in Alzheimer's diseases, the Lewy bodies in Parkinson's disease, the round and skein inclusions in amyotrophic lateral sclerosis, represent an extrema ratio of the cells to limit the damages of these undesired oligomers, the choice by the cell of the lesser of two evils: the small reactive oligomers and the large inert deposits, that become unable to be cleared with aging or disease progression.

\section{AUTHOR CONTRIBUTIONS}

FC and BM designed the work, performed literature search, wrote the manuscript and critically revised it.

Beeg, M., Stravalaci, M., Romeo, M., Carrá, A. D., Cagnotto, A., Rossi, A., et al. (2016). Clusterin binds to A $\beta 1-42$ oligomers with high affinity and interferes with peptide aggregation by inhibiting primary and secondary nucleation. J. Biol. Chem. 291, 6958-6966. doi: 10.1074/jbc.M115.689539

Bemporad, F., and Chiti, F. (2012). Protein misfolded oligomers: experimental approaches, mechanism of formation, and structure-toxicity relationships. Chem. Biol. 19, 315-327. doi: 10.1016/j.chembiol.2012.02.003

Benoit, M. E., Hernandez, M. X., Dinh, M. L., Benavente, F., Vasquez, O., and Tenner, A. J. (2013). C1q-induced LRP1B and GPR6 proteins expressed early in 
Alzheimer disease mouse models, are essential for the C1q-mediated protection against amyloid- $\beta$ neurotoxicity. J. Biol. Chem. 288, 654-665. doi: 10.1074/jbc. M112.400168

Binger, K. J., Ecroyd, H., Yang, S., Carver, J. A., Howlett, G. J., and Griffin, M. D. W. (2013). Avoiding the oligomeric state: $\alpha B$-crystallin inhibits fragmentation and induces dissociation of apolipoprotein C-II amyloid fibrils. FASEB J. 27, 1214-1222. doi: 10.1096/fj.12-220657

Bolognesi, B., Kumita, J. R., Barros, T. P., Esbjorner, E. K., Luheshi, L. M., Crowther, D. C., et al. (2010). ANS binding reveals common features of cytotoxic amyloid species. ACS Chem. Biol. 5, 735-740. doi: 10.1021/cb10 01203

Brehme, M., Voisine, C., Rolland, T., Wachi, S., Soper, J. H., Zhu, Y., et al. (2014). A chaperome subnetwork safeguards proteostasis in aging and neurodegenerative disease. Cell Rep. 9, 1135-1150. doi: 10.1016/j.celrep.2014.09.042

Campioni, S., Mannini, B., Zampagni, M., Pensalfini, A., Parrini, C., Evangelisti, E., et al. (2010). A causative link between the structure of aberrant protein oligomers and their toxicity. Nat. Chem. Biol. 6, 140-147. doi: $10.1038 /$ nchembio. 283

Cappelli, S., Penco, A., Mannini, B., Cascella, R., Wilson, M., Ecroyd, H., et al. (2016). Effect of molecular chaperones on aberrant protein oligomers in vitro: super- versus sub-stoichiometric chaperone concentrations. Biol. Chem. 397, 401-415. doi: 10.1515/hsz-2015-0250

Carrotta, R., Canale, C., Diaspro, A., Trapani, A., Biagio, P. L., and Bulone, D. (2012). Inhibiting effect of $\alpha_{s 1}$-casein on $A \beta_{1-40}$ fibrillogenesis. Biochim. Biophys. Acta 1820, 124-132. doi: 10.1016/j.bbagen.2011.11.010

Cascella, R., Conti, S., Mannini, B., Li, X., Buxbaum, J. N., Tiribilli, B., et al. (2013). Transthyretin suppresses the toxicity of oligomers formed by misfolded proteins in vitro. Biochim. Biophys. Acta 1832, 2302-2314. doi: 10.1016/j. bbadis.2013.09.011

Castellano, L. M., Bart, S. M., Holmes, V. M., Weissman, D., and Shorter, J. (2015). Repurposing Hsp104 to antagonize seminal amyloid and counter HIV infection. Chem. Biol. 22, 1074-1086. doi: 10.1016/j.chembiol.2015. 07.007

Cheng, J. S., Dubal, D. B., Kim, D. H., Legleiter, J., Cheng, I. H., Yu, G. Q., et al. (2009). Collagen VI protects neurons against A $\beta$ toxicity. Nat. Neurosci. 12, 119-121. doi: 10.1038/nn.2240

Cizas, P., Budvytyte, R., Morkuniene, R., Moldovan, R., Broccio, M., Lösche, M., et al. (2010). Size-dependent neurotoxicity of $\beta$-amyloid oligomers. Arch. Biochem. Biophys. 496, 84-92. doi: 10.1016/j.abb.2010.02.001

Cohen, S. I. A., Arosio, P., Presto, J., Kurudenkandy, F. R., Biverstål, H., Dolfe, L., et al. (2015). A molecular chaperone breaks the catalytic cycle that generates toxic A $\beta$ oligomers. Nat. Struct. Mol. Biol. 22, 207-213. doi: 10.1038/nsmb.2971

Fernandez-Funez, P., Sanchez-Garcia, J., de Mena, L., Zhang, Y., Levites, Y., Khare, S., et al. (2016). Holdase activity of secreted Hsp70 masks amyloid$\beta 42$ neurotoxicity in Drosophila. Proc. Natl. Acad. Sci. US A 113, E5212-E5221. doi: 10.1073/pnas. 1608045113

Hyttinen, J. M. T., Amadio, M., Viiri, J., Pascale, A., Salminen, A., and Kaarniranta, K. (2014). Clearance of misfolded and aggregated proteins by aggrephagy and implications for aggregation diseases. Ageing Res. Rev. 18, 16-28. doi: 10.1016/j.arr.2014.07.002

Jia, B., Wu, Y., and Zhou, Y. (2014). 14-3-3 and aggresome formation: implications in neurodegenerative diseases. Prion 8, 173-177. doi: 10.4161/ pri.28123

Johnston, J. A., Ward, C. L., and Kopito, R. R. (1998). A cellular response to misfolded proteins aggresomes. J. Cell Biol. 143, 1883-1898. doi: 10.1083/ jcb.143.7.1883

Kaushik, S., and Cuervo, A. M. (2012). Chaperones in autophagy. Pharmacol. Res. 66, 484-493. doi: 10.1016/j.phrs.2012.10.002

Kawaguchi, Y., Kovacs, J. J., McLaurin, A., Vance, J. M., Ito, A., and Yao, T. P. (2003). The deacetylase HDAC6 regulates aggresome formation and cell viability in response to misfolded protein stress. Cell 115, 727-738. doi: 10.1016/s0092-8674(03)00939-5

Knowles, T. P. J., Shu, W., Devlin, G. L., Meehan, S., Auer, S., Dobson, C. M., et al. (2007). Kinetics and thermodynamics of amyloid formation from direct measurements of fluctuations in fibril mass. Proc. Natl. Acad. Sci. U S A 104, 10016-10021. doi: 10.1073/pnas.0610659104

Labbadia, J., and Morimoto, R. I. (2015). The biology of proteostasis in aging and disease. Annu. Rev. Biochem. 84, 435-464. doi: 10.1146/annurev-biochem060614-033955
Li, X., Masliah, E., Reixach, N., and Buxbaum, J. N. (2011). Neuronal production of transthyretin in human and murine Alzheimer's disease: is it protective? J. Neurosci. 31, 12483-12490. doi: 10.1523/JNEUROSCI.241711.2011

Li, X., Zhang, X., Ladiwala, A. R. A., Du, D., Yadav, J. K., Tessier, P. M., et al. (2013). Mechanisms of transthyretin inhibition of $\beta$-amyloid aggregation in vitro. J. Neurosci. 33, 19423-19433. doi: 10.1523/JNEUROSCI.256113.2013

Mannini, B., Cascella, R., Zampagni, M., van Waarde-Verhagen, M., Meehan, S., Roodveldt, C., et al. (2012). Molecular mechanisms used by chaperones to reduce the toxicity of aberrant protein oligomers. Proc. Natl. Acad. Sci. U S A 109, 12479-12484. doi: 10.1073/pnas.1117799109

Mannini, B., Mulvihill, E., Sgromo, C., Cascella, R., Khodarahmi, R., Ramazzotti, M., et al. (2014). Toxicity of protein oligomers is rationalized by a function combining size and surface hydrophobicity. ACS Chem. Biol. 9, 2309-2317. doi: 10.1021/cb500505m

Månsson, C., Arosio, P., Hussein, R., Kampinga, H. H., Hashem, R. M., Boelens, W. C., et al. (2014). Interaction of the molecular chaperone DNAJB6 with growing amyloid- $\beta 42$ (A $\beta 42)$ aggregates leads to sub-stoichiometric inhibition of amyloid formation. J. Biol. Chem. 289, 31066-31076. doi: 10.1074/jbc.M114.595124

Miller, S. B. M., Mogk, A., and Bukau, B. (2015). Spatially organized aggregation of misfolded proteins as cellular stress defense strategy. J. Mol. Biol. 427, 1564-1574. doi: 10.1016/j.jmb.2015.02.006

Mogk, A., Kummer, E., and Bukau, B. (2015). Cooperation of Hsp70 and Hsp100 chaperone machines in protein disaggregation. Front. Mol. Biosci. 2:22. doi: 10.3389/fmolb.2015.00022

Moll, L., Ben-Gedalya, T., Reuveni, H., and Cohen, E. (2016). The inhibition of IGF-1 signaling promotes proteostasis by enhancing protein aggregation and deposition. FASEB J. 30, 1659-1669. doi: 10.1096/fj.15-281675

Narayan, P., Meehan, S., Carver, J. A., Wilson, M. R., Dobson, C. M., and Klenerman, D. (2012). Amyloid- $\beta$ oligomers are sequestered by both intracellular and extracellular chaperones. Biochemistry 51, 9270-9276. doi: $10.1021 /$ bi301277k

Narayan, P., Orte, A., Clarke, R. W., Bolognesi, B., Hook, S., Ganzinger, K. A., et al. (2011). The extracellular chaperone clusterin sequesters oligomeric forms of the amyloid- $\beta_{1-40}$ peptide. Nat. Struct. Mol. Biol. 19, 79-83. doi: 10.1038/ nsmb. 2191

Nillegoda, N. B., and Bukau, B. (2015). Metazoan Hsp70-based protein disaggregases: emergence and mechanisms. Front. Mol. Biosci. 2:57. doi: $10.3389 /$ fmolb.2015.00057

Nillegoda, N. B., Kirstein, J., Szlachcic, A., Berynskyy, M., Stank, A., Stengel, F., et al. (2015). Crucial HSP70 co-chaperone complex unlocks metazoan protein disaggregation. Nature 524, 247-251. doi: 10.1038/nature14884

Ojha, J., Masilamoni, G., Dunlap, D., Udoff, R. A., and Cashikar, A. G. (2011). Sequestration of toxic oligomers by HspB1 as a cytoprotective mechanism. Mol. Cell. Biol. 31, 3146-3157. doi: 10.1128/MCB.01187-10

Olzscha, H., Schermann, S. M., Woerner, A. C., Pinkert, S., Hecht, M. H., Tartaglia, G. G., et al. (2011). Amyloid-like aggregates sequester numerous metastable proteins with essential cellular functions. Cell 144, 67-78. doi: 10.1016/j.cell.2010.11.050

Ouyang, H., Ali, Y. O., Ravichandran, M., Dong, A., Qiu, W., MacKenzie, F., et al (2012). Protein aggregates are recruited to aggresome by histone deacetylase 6 via unanchored ubiquitin C termini. J. Biol. Chem. 287, 2317-2327. doi: 10.1074/jbc.M111.273730

Raman, B., Ban, T., Sakai, M., Pasta, S. Y., Ramakrishna, T., Naiki, H., et al. (2005). $\alpha \mathrm{B}$-crystallin, a small heat-shock protein, prevents the amyloid fibril growth of an amyloid $\beta$-peptide and $\beta 2$-microglobulin. Biochem. J. 392, 573-581. doi: 10.1042/bj20050339

Shammas, S. L., Waudby, C. A., Wang, S., Buell, A. K., Knowles, T. P. J., Ecroyd, H., et al. (2011). Binding of the molecular chaperone $\alpha \mathrm{b}$-crystallin to $\mathrm{A} \beta$ amyloid fibrils inhibits fibril elongation. Biophys. J. 101, 1681-1689. doi: 10.1016/j.bpj. 2011.07.056

Sharoar, M. G., Shahnawaz, M., Islam, M. I., Ramasamy, V. S., Shin, S. Y., and Park, I. S. (2013). The inhibitory effects of Escherichia coli maltose binding protein on $\beta$-amyloid aggregation and cytotoxicity. Arch. Biochem. Biophys. 538, 41-48. doi: 10.1016/j.abb.2013.08.004

Shinohara, H., Inaguma, Y., Goto, S., Inagaki, T., and Kato, K. (1993). $\alpha$ B crystallin and HSP28 are enhanced in the cerebral cortex of patients with 
Alzheimer's disease. J. Neurol. Sci. 119, 203-208. doi: 10.1016/0022-510x(93) 90135-1

Sörgjerd, K. M., Zako, T., Sakono, M., Stirling, P. C., Leroux, M. R., Saito, T., et al. (2013). Human prefoldin inhibits amyloid- $\beta$ (A $\beta$ ) fibrillation and contributes to formation of nontoxic A $\beta$ aggregates. Biochemistry 52, 3532-3542. doi: 10.1021/bi301705c

Villaverde, A., and Carrió, M. M. (2003). Protein aggregation in recombinant bacteria: biological role of inclusion bodies. Biotechnol. Lett. 25, 1385-1395. doi: 10.1023/A:1025024104862

Wakabayashi, K., Tanji, K., Mori, F., and Takahashi, H. (2007). The lewy body in Parkinson's disease: molecules implicated in the formation and degradation of $\alpha$-synuclein aggregates. Neuropathology 27, 494-506. doi: 10.1111/j.1440-1789. 2007.00803.x

Walther, D. M., Kasturi, P., Zheng, M., Pinkert, S., Vecchi, G., Ciryam, P., et al. (2015). Widespread proteome remodeling and aggregation in aging C. elegans. Cell 161, 919-932. doi: 10.1016/j.cell.2015.03.032

Waudby, C. A., Knowles, T. P. J., Devlin, G. L., Skepper, J. N., Ecroyd, H., Carver, J. A., et al. (2010). The interaction of $\alpha \mathrm{B}$-crystallin with mature $\alpha$-synuclein amyloid fibrils inhibits their elongation. Biophys. J. 98, 843-851. doi: 10.1016/j.bpj.2009.10.056

Xu, Z., Graham, K., Foote, M., Liang, F., Rizkallah, R., Hurt, M., et al. (2013). 14-33 protein targets misfolded chaperone-associated proteins to aggresomes. J. Cell Sci. 126, 4173-4186. doi: 10.1242/jcs. 126102
Xu, L., Wu, S., Buell, A. K., Cohen, S. I. A., Chen, L., Hu, W., et al. (2013). Influence of specific HSP70 domains on fibril formation of the yeast prion protein Ure2. Philos. Trans. R. Soc. Lond. B Biol. Sci. 368:20110410. doi: 10.1098/ rstb.2011.0410

Yerbury, J. J., Gower, D., Vanags, L., Roberts, K., Lee, J. A., and Ecroyd, H. (2013). The small heat shock proteins $\alpha \mathrm{B}$-crystallin and Hsp27 suppress SOD1 aggregation in vitro. Cell Stress Chaperones 18, 251-257. doi: 10.1007/s12192-012-0371-1

Yerbury, J. J., Poon, S., Meehan, S., Thompson, B., Kumita, J. R., Dobson, C. M., et al. (2007). The extracellular chaperone clusterin influences amyloid formation and toxicity by interacting with prefibrillar structures. FASEB J. 21, 2312-2322. doi: 10.1096/fj.06-7986com

Conflict of Interest Statement: The authors declare that the research was conducted in the absence of any commercial or financial relationships that could be construed as a potential conflict of interest.

Copyright (c) 2017 Mannini and Chiti. This is an open-access article distributed under the terms of the Creative Commons Attribution License (CC BY). The use, distribution or reproduction in other forums is permitted, provided the original author(s) or licensor are credited and that the original publication in this journal is cited, in accordance with accepted academic practice. No use, distribution or reproduction is permitted which does not comply with these terms. 